\title{
Giant Solitary Forearm Exostosis in A Child
}

\author{
WI Faisham, M Med Ortho, W Zulmi, M S Ortho \\ Department of Orthopaedics, School of Medical Science Universiti Sains Malaysia, Kota Bahru, Malaysia
}

\begin{abstract}
Forearm deformity secondary to giant solitary ulna exostosis is rare. We describe a rare presentation of symptomatic solitary giant exostosis involving the entire distal ulna resulting in ulnar bowing of the forearm in a five-year-old boy. The tumour was completely resected and the defect was reconstructed with an allograft wrapped with a free autogenous periosteal tubular sleeve to deliver fresh pluripotential cells for better incorporation and integration. The distal ulna physes was preserved. An osteotomy was performed on the radius to correct the deformity. One year after surgery, the deformity remains corrected with normal bone length and excellent hand function. There is no evidence of local recurrence and the allograft has fully incorporated.
\end{abstract}

Key Words:

Distal ulna exostosis, resection, Allograft, and periosteum sleeve

\section{INTRODUCTION}

Solitary exostosis is the most common benign bone tumour, and consists of a cartilage-capped bony projection on the external surface of a bone. It is not considered a true neoplasm, rather it is a hamartoma produced by the growth of subperiosteal aberrant foci of cartilage. It is asymptomatic in the majority of people and is typically incidentally discovered around the age of puberty. We describe here a rare presentation of symptomatic solitary giant exostosis, involving the entire distal ulna resulting in a forearm deformity in a five-year-old child. Following is a description of ensuing surgical management and the subsequent outcome.

\section{CASE REPORT}

A five-year old boy presented in our institution with progressive swelling of the right forearm and deformity developing over a period of two years. The swelling was first noticed at the age of three and was asymptomatic. The family noticed that it was growing rapidly for the past six month and the patient consistently complained of tightness and weakness of the hand. Physical examination revealed a bony hard mass extending from the mid-shaft to the distal of the ulna, associated with ulnar bowing of the forearm. Examination revealed no evidence of neurological compromise in the hand and both the ulna and radial arterial pulses were palpable (Figure 1).

Plain radiographic examination showed a fusiform bony lesion at the meta-diaphyseal region of the right ulna. The distal ulna physis was still intact and preserved. Chronic compression by the lesion caused thinning of the radial cortex and ulnar deviation of the radius. In view of the symptoms and progressive deformity we performed a resection of the entire lesion. Intra-operatively, the ulna neurovascular structure was preserved. The distal ulna physeal region was not involved and thus maintained. The four centimetre long osseous defect was reconstructed with deep frozen ulna allograft to reduced donor site morbidity in growing bone. A non-vascularised periosteal flap taken from the tibia was wrapped around the entire allograft and host junction to augment incorporation. The radial deformity was corrected by a long oblique osteotomy. The reconstruction and the distal radio-ulna joint were stabilized with smooth wires (Figure 2).
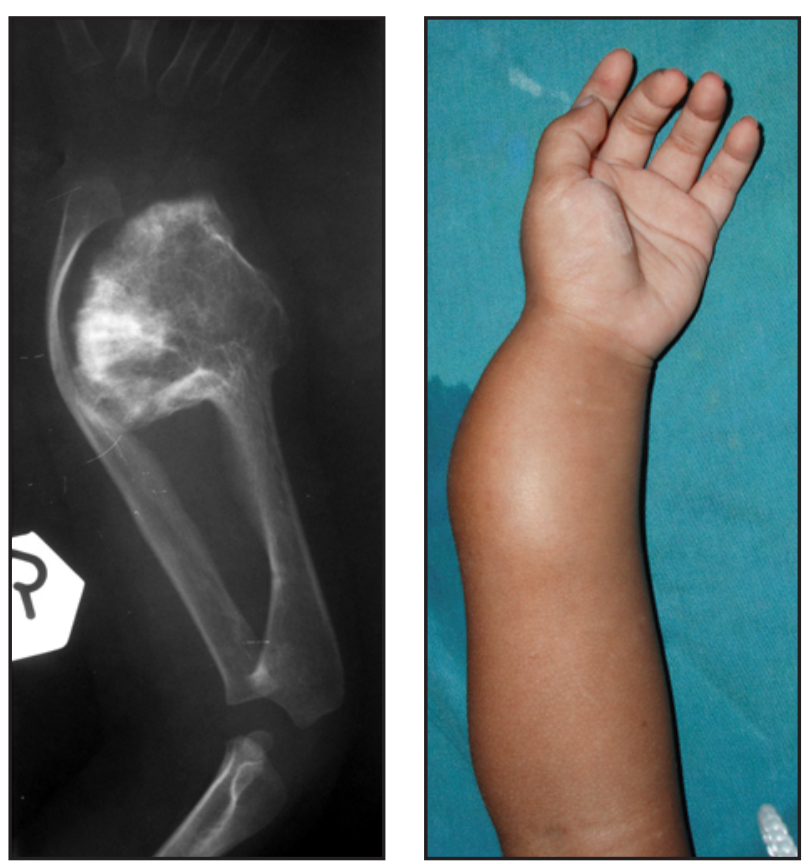

Fig. 1: Giant exostosis involving the entire distal ulna with ulna bowing of the radius.

Corresponding Author: WI Faisham, Department of Orthopaedic, School of Medical Science, Universiti Sains Malaysia, 16150 Kubang Kerian, Kelantan, Malaysia Email: faisham@kb.usm.my 


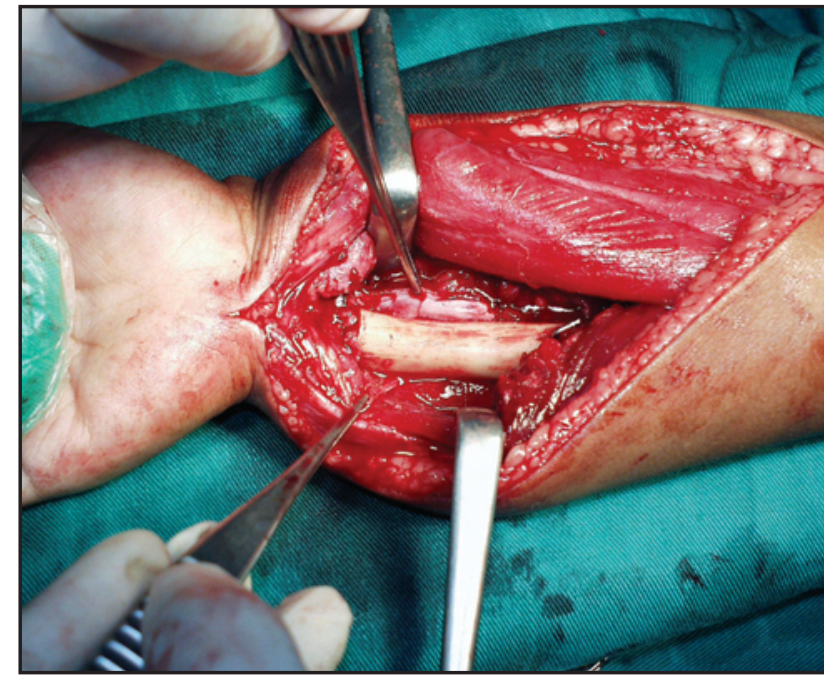

Fig. 2A: The ulna exostosis was resected with preservation of the distal ulna physis and reconstruction with allograft.

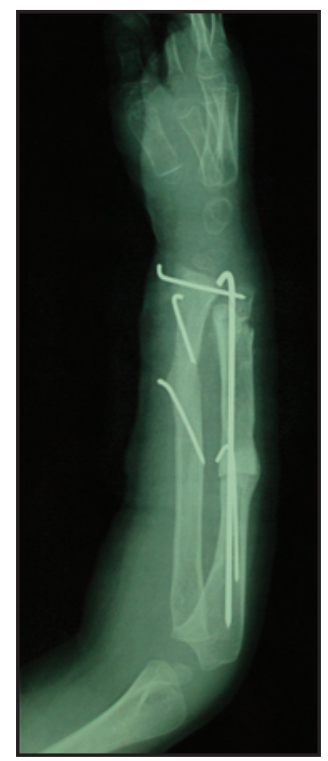

Fig. 2C: The radiograph showed bony union at host-allograft junction, and correction of the deformity (after four months).

Post-operatively the forearm was protected in a full-length cast for six weeks. The distal radial-ulna wire was removed at two months post-surgery. One year after surgery the patient was asymptomatic. The deformity was corrected and wrist and hand function was good, with $80^{\circ}$ wrist palmar flexion and $30^{\circ}$ dorsiflexion and the hand grip was full with power of MRC grade 4/5. Radial and ulna deviation were $30^{\circ}$, however forearm supination and pronation were limited to $40^{\circ}$. There was no morbidity at the periosteal donor site. There was no evidence of local recurrence and the allograft had fully incorporated. Bone scan at one year showed increased uptake of the entire allograft (Figure 3).

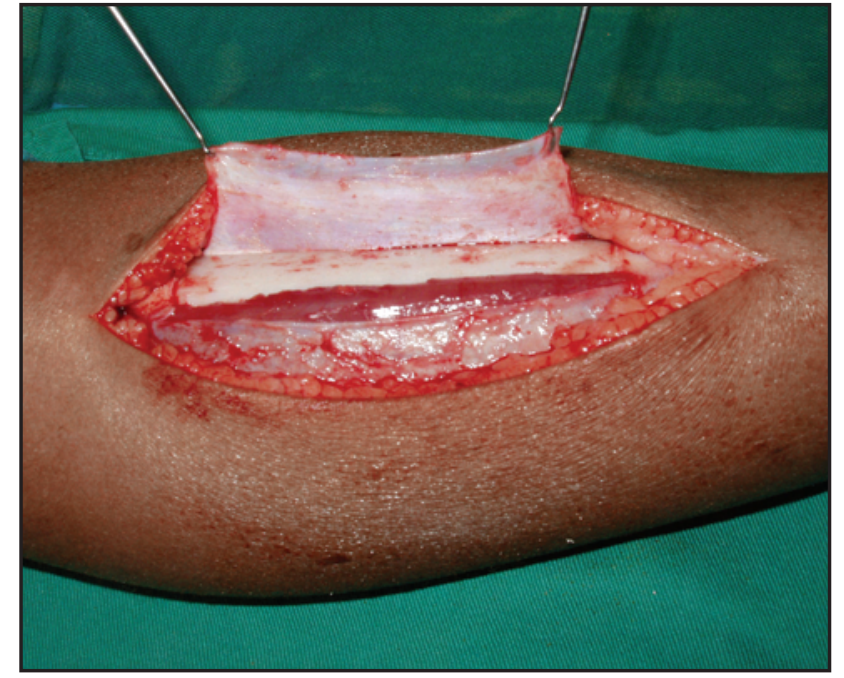

Fig. 2B: Periosteal flap from the tibia was wrapped around the allograft to enhanced healing.

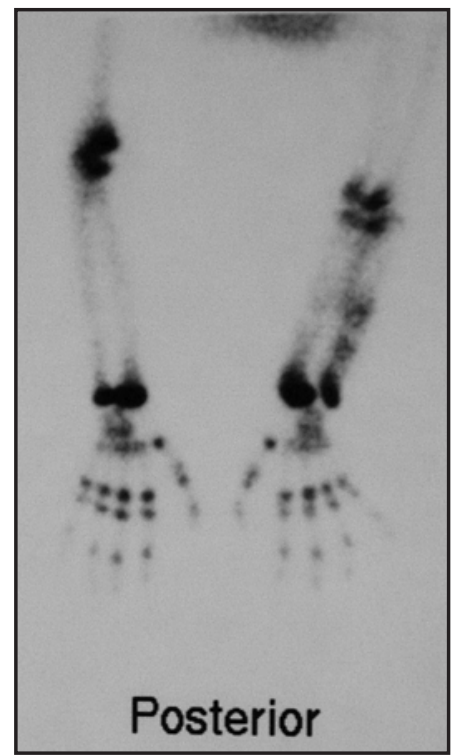

Fig. 3: Bone scan at one year revealed significant uptake at ulna allograft junction consistent with viability.

\section{DISCUSSION}

Deformity of the forearm is commonly observed in multiple hereditary exostoses, and is due to defective metaphyseal remodelling and asymmetrical retardation of longitudinal bone growth ${ }^{1,2}$. The most common deformity is a combination of relative shortening of the ulna, bowing of one or both forearm bones, ulnar tilt of the distal epiphysis of the radius, ulnar deviation of the hand, progressive translocation towards the ulna of the carpus and dislocation of the radial head ${ }^{1,2}$. A solitary isolated giant fusiform forearm exostosis presenting in early childhood is rare. The progressive enlargement of the lesion and local compressive effect to adjacent bone lead to deformity, thus complicating surgical 
management. Furthermore, the large swelling caused chronic compressive neuropathy and interfered with gliding motion of the tendons resulting in poor hand function.

In the past, the operative management had to be delayed until puberty because of the unfavourable results of early intervention. In recent years, early operative intervention was performed to prevent or reduce the progression of deformity, hence the unacceptable frequency of residual functional impairment, particularly with regard to radial head dislocation ${ }^{1,2}$. Partial resection of the fusiform exostosis is reported to have a high recurrence rate and may necessitate repeated surgical interventions. Moreover, the progression of deformity cannot be predicted. On the other hand, resection of the entire lesion leads to a massive osseous defect and subsequent disturbed growth and function. A deformity caused by a solitary giant osteochondroma should be managed differently from multiple exostoses, as the remaining physeal growth plate is not involved. To overcome this problem, we describe a procedure involving total resection of the lesion with preservation of distal ulna physeal growth plate and reconstruction of the resultant defect with allograft.
Reconstruction of a long segment osseous defect by a strut graft of autogenous bone is a standard practice in adults, but in paediatric donor sites, morbidity poses a major problem with the possibility of permanent deformity and disability. Allograft is therefore a good alternative; however, bony incorporation could be delayed and there is subsequent risk of fracture and dissolution of the graft. We used allograft wrapped with the autogenous periosteal flap to deliver fresh pluripotential cell for better incorporation and integration ${ }^{3,4}$. Allograft healing and strength can be achieved relatively early thereby reducing the risk of allograft complications ${ }^{3,4}$. In this case, radiological evidence of bony incorporation and union was seen at six months. Furthermore, the remaining physeal plate of the distal ulna was functioning giving evidence of proper longitudinal growth and forearm alignment.

This method of treatment allows rapid functional recovery without sacrificing the growth potential of a long bone. It can be considered for other conditions with long bone defect not involving the epiphysis. 


\section{REFERENCES}

1. Ip D, Li YH, Chow W, Leong JC. Reconstruction of forearm deformity in multiple cartilagenous exostoses. J Pediatr Orthop 2003; 12(1):17-21.

2. Peterson HA. Deformity and problem of the forearm in children with multiple hereditary osteochondromata. J Pediatr Orthop 1994; 13: 92-100.

3. Karaoglu S, Baktir A, Kabak S, Arasi H. Experimental repair of segmental bone defect in rabbit by demineralised allograft covered by free autogenous periosteum. Injury 2002; 33(8): 679-83.

4. Neel M. The use of a periosteal membrane for bone graft containment at allograft host junction after tumour resection and reconstruction with bulk allograft. Orthopedics 2003; 26(5): 587-9. 